\title{
ASPECTOS LÚDICOS DE LO COTIDIANO EN EL ARTE PALEOLÍTICO
}

\section{LUDIC ASPECTS OF THE EVERYDAY IN PALEOLITHIC ART}

\author{
Alberto Lombo Montañés \\ Universidad de Zaragoza \\ albertolommon@hotmail.com \\ ORCID: 0000-0003-0972-2459
}

DOI: $10.1387 /$ veleia.18073

\begin{abstract}
Resumen: Las sonrisas, risas y caricaturas que se han detectado en grafías humanas del arte paleolítico permiten investigar aspectos lúdicos de lo cotidiano en la Prehistoria. La definición, clasificación y análisis de estas grafías demuestran que este fenómeno lúdico, basado en la exageración de las formas y las expresiones alegres, se manifestó en diversas zonas de la región francesa durante el Magdaleniense. Estos documentos son interpretados como la prueba gráfica de un sentido del humor prehistórico.
\end{abstract}

Palabras clave: Sonrisas; risas; caricaturas; antropomorfos; arte paleolítico; Magdaleniense; sudoeste de Europa.

Abstract: Smiles, laughter and caricatures detected on certain Palaeolithic human representations permit us to draw some conclusions about the ludic component of everyday life in Prehistory. This paper offers a definition, classification and analysis of the selected motives and suggests that this playful phenomenon, based on exaggerated forms and cheerful expressions, was developed on different regions in present-day France during the Magdalenian period. These documents provide the graphic proof of the existence of a real prehistoric sense of humour.

Keywords: Smiles; Laughs; Caricatures; Anthropomorphic figure; Palaeolithic Art; Magdalenian; South-Western Europe.

Recibido:16-02-2017

Informado: 24-04-2017

Definitivo: 11-05-2017

La sonrisa, la risa y las caricaturas son fenómenos lúdicos propios del ámbito cotidiano. Todas las sociedades humanas reflejan de forma gráfica sus diversiones cotidianas y los grupos paleolíticos no han sido una excepción. En el arte paleolítico se documentan $81 \mathrm{UG}$ (unidades gráficas) que pueden catalogarse como sonrisas, risas y caricaturas (fig. 1). Se trata de un número reducido de grafías humanas dentro del cómputo general de representaciones antropomorfas del arte paleolítico europeo $^{1}$.

1 En total podemos contabilizar 1147 UG humanas en el arte paleolítico europeo, de las cuales sólo un
$7 \%$ pueden calificarse de caricaturas, risas y sonrisas (Lombo 2015b, 208). 


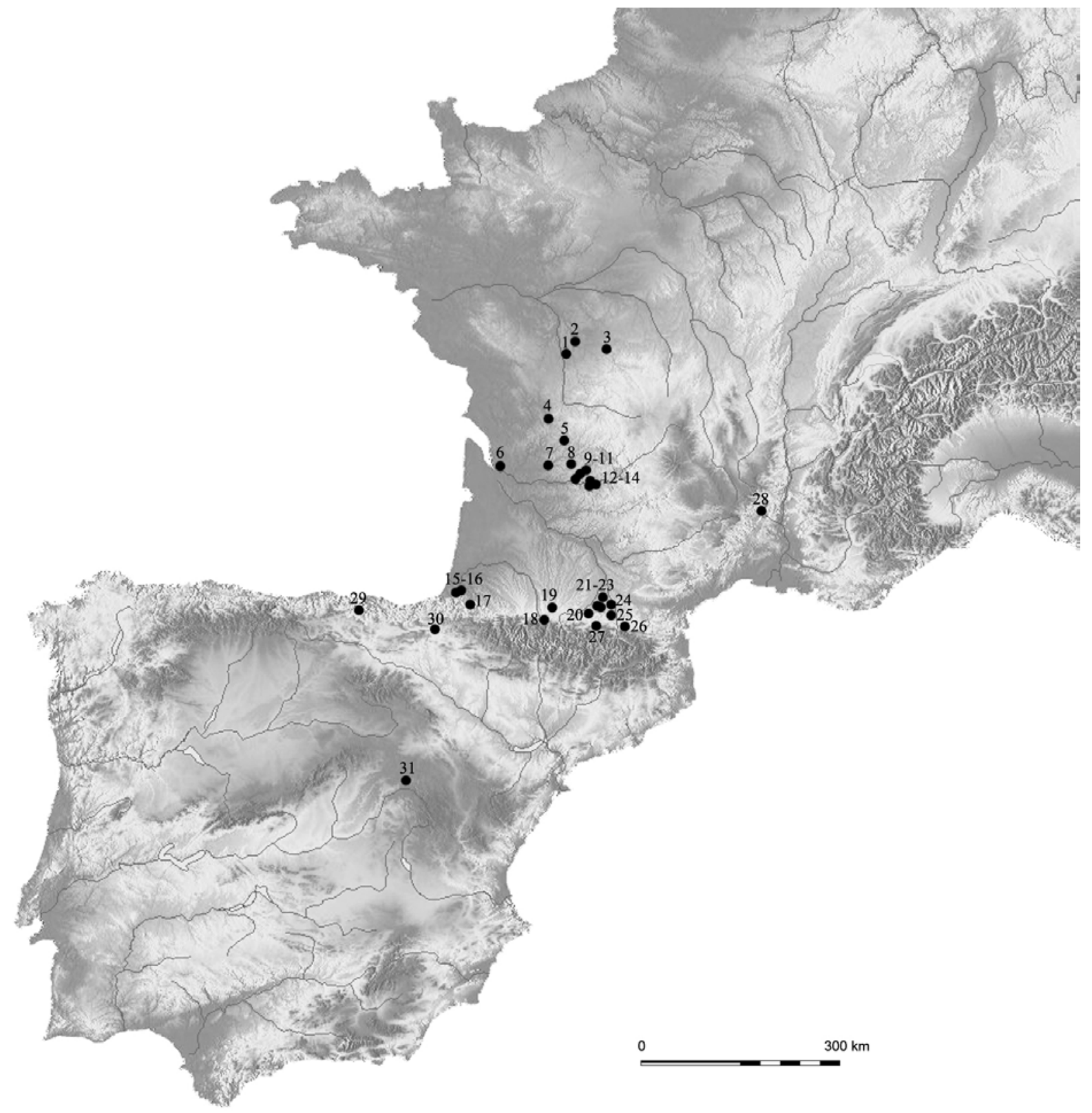

Figura I. Yacimientos con caricaturas, risas y sonrisas. 1 La Marche; 2 Bourdois; 3 La Garenne; 4 Le Placard; 5 Peyzie; 6 Roc de Marcamps; 7 Le Gabillou; 8 Rouffignac; 9 Saint-Cirq; 10 Laugerie-Basse; 11 La Madeleine; 12 Péchialet; 13 Combarelles; 14 Bernifal; 15 Isturitz; 16 Erberua; 17 Sinihikole-Ko-Karbia; 18 Labastide; 19 Gourdan; 20 Marsoulas; 21 Trois-Frères; 22 Enlène; 23 Mas d'Azil; 24 Le Portel; 25 Bédeilhac; 26 Fontantet; 27 Ker de Massat; 28 Colombier; 29 Juan Gómez; 30 Abauntz; 31 Casares 


\section{Por unA Nueva FORMA DE ENTENDER LO LÚdico}

La teoría lúdica que algunos historiadores atribuyen a los arqueólogos decimonónicos Lartet, Christy, Piette y Mortillet (Richard 1993; Sinués 2010) tiene su origen en las ideas de Schiller (1981, carta 12). Estas sobreviven en el siglo xx (Verworn 1907; Forrer 1908; Ridell 1940) y muy parcialmente en Luquet $(1926,133)$, y han sido más recientemente sostenidas por Halvestron (1987). En esta teoría lo lúdico se vincula con el ocio, la vida idílica, lo simple, lo espontáneo, lo inocente, lo infantil, lo intrascendente, lo pagano, lo imitativo y lo decorativo, para considerar, en definitiva, el arte paleolítico como un arte menor o sin significado (Fraguas Bravo 2007, 24; Fiore 2014, 437; Hernando 2014, 10; Lombo 2015a, 8-9; Sinués 2010, 83-84). Aparte de esta teoría, y durante todo el siglo xx, se hicieron breves apuntes acerca de la risa o la sonrisa (Pales, SaintPéreuse 1976, pl. 82, pl. 65; Barrière 1982, 142; Roussot 1986, 97; Vialou 1986, 255; Duhard 1992, 137; 1996, 208; Iakovleva, Pinçon 1997, 82 y 138; Delluc, Delluc 2009, 659) y las caricaturas (Hamy 1908, 394; Luquet 1910, 413; Hernández-Pacheco 1919, 222; Capitan et al. 1924, 53, 95 y 113; Saint-Périer 1936, 15; Cabré 1940-41, 95; Ripoll 1957-58, 167; Patte 1960, 76; Pales, Tassin de Saint Péreuse 1976, 7 y 50; Gailli 1980, 33; Vialou 1986, 344; Clottes 1986-87, 45; Bahn, Vertut 1989, 136; Leonardi 1989, 181; Alpert 1992, 231; Tymula 1995, 224; Duhard 1996, 19, 43, 57 y 139; Plassard 1999, 73; Airvaux 2001, 92; Sanchidrián 2001, 223; Delluc, Delluc 2009, 659; Fuentes 2010, 385 y 390)².

Es preciso examinar las referencias a la risa, la sonrisa y las caricaturas surgidas al margen de la teoría lúdica ${ }^{3}$. Este tipo de apreciaciones — que se han mantenido dispersas e inconexas en la bibliografía - no han tenido apenas repercusión en los estudios de arte paleolítico. Esto es así, porque el paradigma interpretativo, desde Breuil (Cartailhac, Breuil 1906, 85) a Leroi-Gourhan (1984, 608-609), está basado en una idea de lo sagrado que no contempla el humor (Lombo 2013; 2015 b , 87). Una nueva forma de entender lo cómico debería superar la falsa dicotomía que contrapone lo lúdico (pagano) considerado como lo simple, intrascendente o "el arte por el arte», con lo religioso (sagrado), complejo, trascendente o "arte funcional», ya que este dualismo no parece operar en los pueblos sin escritura (Evans-Pritchard 1988, 109).

Cabe recordar que la Prehistoria se ha configurado como disciplina científica en base a los postulados de las ciencias naturales. Por lo tanto el origen de la Prehistoria, el método arqueológico e incluso la clasificación de los objetos son deudores de las ciencias naturales; sin embargo el objetivo de la Prehistoria no son esos objetos, sino las sociedades que los produjeron, es decir, el objetivo fundamental de la Prehistoria pertenece por entero a las ciencias humanas (Beaune 2007). Esta enorme paradoja, que ha causado y sigue causando mucha confusión en nuestra disciplina, es fundamental para

\footnotetext{
2 Son casi siempre descripciones de cabezas humanas con rasgos anatómicos exagerados y breves comentarios acerca de su sentido humorístico. Por ejemplo Gailli (1980, 33) describe una de las cabezas humanas de Ker de Massat como una «véritable caricature, digne de nos meilleurs humoristes»; los Delluc $(2009,659)$ afirman que algunas cabezas humanas del Périgord «semblent avoir été délibérément dessinées pour surprendre voire faire sourire»; Pales y Saint Péreuse $(1976,50)$ observan en las grafías de la Marche «...une pointe d'ironie destinée à provoquer au moins le sourire. On reviendrait ainsi à cette humour des Préhistoriques, de ceux de La Marche entre tous qui, non
}

contents d'être des non-conformistes auraient été de surcroît des précurseurs»; y Plassard (1999, 73 y 71) encuentra en Rouffignac un auténtico diseño en "caricatura», una muestra de «la discrète finesse de l'humour paléolithique».

3 Es conveniente no confundirlas. La llamada teoría lúdica pretende reducir la variabilidad gráfica a una sola explicación. Como acertadamente advierte Huizinga (1968, 199), sería demasiado osado concebir las pinturas rupestres como el producto de un impulso lúdico congénito. Necesitamos un concepto de lo lúdico que se desvincule de los tópicos precedentes y sea compatible con otras interpretaciones. 
entender por qué los prehistoriadores no han estudiado a fondo el tema del humor. Pues el estudio del humor tiene una larga tradición en las ciencias humanas, pero es reciente en la ciencia auxiliar de la Prehistoria. Además, la Antropología ha privilegiado siempre los aspectos exóticos a los cotidianos (Evans-Pritchard 1988, 21), dando una visión demasiado esotérica (seria) de la religiones de los pueblos sin escritura, e ignorando, hasta hace unos años, los temas relativos al humor (Driessen 1999).

El objetivo de este trabajo es reunir, clasificar y analizar los mencionados documentos gráficos, además de reflexionar sobre el alcance de lo que podrían ser las primeras expresiones gráficas del humor conocidas.

\section{Estado de la Cuestión y mÉtodo}

La evolución de risa y la sonrisa tiene dos momentos cruciales:

a) la risa o la sonrisa antes de la aparición del lenguaje (periodo pre-lingüístico) como comunicación no-verbal (Darwin 1984, 215) relacionada con el mundo de los sonidos (Davila $e t$ al. 2009) y la interacción auditiva que todavía conservamos (Beaudet 1996) y,

b) la risa o la sonrisa tras la aparición del lenguaje (Dunbar 1996; Provine 2000) cuyas complejidades multiplicaron las dimensiones culturales de la risa (música, danza, canto, chistes, arte).

Los estudios neurocientíficos han resaltado la capacidad del Homo sapiens para percibir complejas incongruencias humorísticas (Mithen 1996, 212) y su reflejo en las expresiones graficas paleolíticas (Guthrie 2005, 372-399; Alpert 2008). Los estudios etnográficos han recogido datos aislados sobre la vida cotidiana de los pueblos ágrafos (Condominas 1991), como el gusto por bromas de índole sexual (Brackelaire 1993, 127), las exhibiciones fálicas (Barley 2010, 108-109) o los chistes obscenos (Lévi-Strauss 1968, 307). Los trabajos antropológicos constatan la función social del humor (Radcliffe-Brown 1940), porque mantiene al grupo unido en momentos adversos (Marshall 1961), refuerza la identidad colectiva y es fundamental en las relaciones intergrupales (Apte 1985, 108; Driessen 1999, 240).

Ahora bien, la investigación del sentido del humor en Prehistoria ha de superar las barreras del léxico establecido. En primer lugar, debe buscar una definición más universal del fenómeno de la caricatura que pueda ser utilizada para todas las épocas y grafías humanas sin exclusión de ninguna ${ }^{4}$. La caricatura se define como una representación, generalmente de la parte superior del cuerpo humano, basada en la exageración y/o síntesis de los rasgos anatómicos con fines alegóricos o humorísticos.

Los siguientes aspectos pueden concebirse como criterios de selección:

a) El formato: la cabeza.

b) El tema: lo humano o lo humano animalizado.

c) La exageración, de rasgos anatómicos concretos como la nariz y el cuello.

d) Formas inventadas, típicas de la caricatura como ojos circulares concéntricos o narices redondas.

Una vez recopilado el repertorio de caricaturas, sonrisas y risas, hemos analizado la distribución geográfica, el soporte, la técnica, la perspectiva, la cronología y en el caso de las caricaturas hemos realizado un estudio morfo-gráfico de las distintas partes de la cabeza humana para ver dónde se concentran las exageraciones anatómicas (Lombo 2015b, 39-42).

${ }^{4}$ Hace unos años nos percatamos que la definición actual de caricatura no refleja la amplitud del fenómeno que describe (Lombo 2013) e iniciamos una investi- gación acerca de su procedimiento gráfico para ahondar en los elementos estructurales que la caracterizan (Lombo 2013, 244-247; 2015b, 31-36). 


\section{ANÁlisis de CARICATURAS, RisAS y SONRISAS}

\section{3.a) Caricaturas}

Las 58 grafías que responden al concepto de caricatura (fig. 2) se dividen en dos tipos: humanas (41 UG) y humanas animalizadas (17 UG) ${ }^{5}$. El tipo humano es predominante en el arte parietal del Midi (11 UG) y contrasta con el arte de las cuevas de la Dordońa, donde casi la mitad de los ejemplos (8 de $17 \mathrm{UG})$ son animalizaciones.

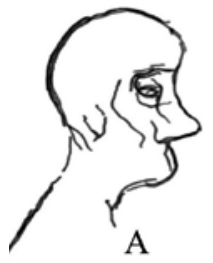

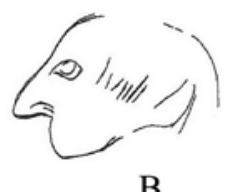

B
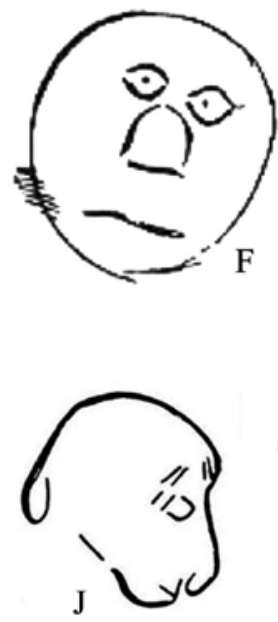
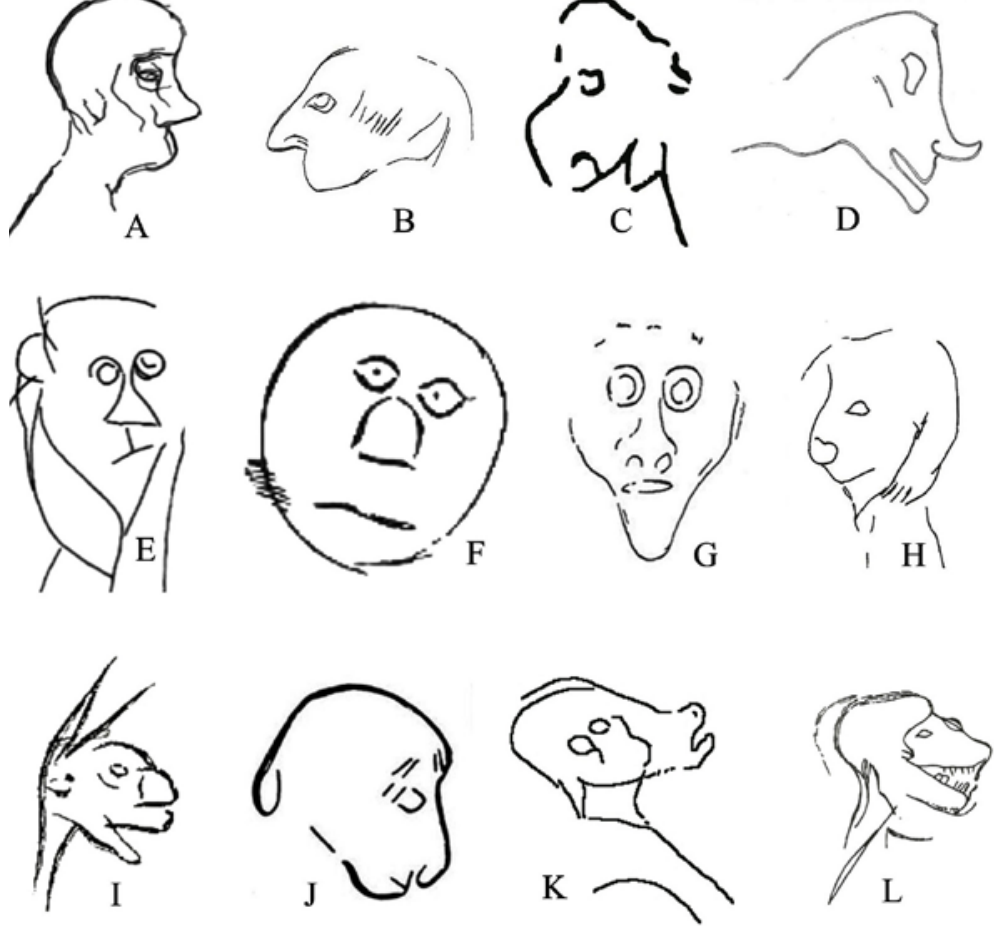

Figura 2. Caricaturas humanas: A La Marche (Pales, Tassin de Saint Péreuse 1976, ob. 12, pl. 24); B La Marche (Mélard 2008, 162, fig. 18); C Fontanet (Clottes et al. 1984, 434, fig. 2); D Rouffignac (Barrière 1982, 142, fig. 43); E Marsoulas (Leroi-Gourhan 1971, 397); F Trois-Frères (Bégouën, Breuil 1958, 35 fig. 39); G Labastide (Omnes 1984, 22, pl. 3); H Combarelles (Barrière 1997, 411, fig. 463). Caricaturas animalizadas: I Le Gabillou (Gaussen 1964, pl. 34, fig. 1); J Saint-Cirq (Delluc et al. 1987, 388, fig. 33); K Trois-Frères (Bégouën et al. 2014, 133, fig. 114); L Ker de Massat (Barrière 1990 35, fig. 15)

5 Cinco de estas caricaturas humanas son sonrientes y dos rientes; sólo una animalizada es riente. Las posibles caricaturas animalizadas tienen un carácter mucho más ambiguo (ver anexo 1). A pesar de las precauciones tomadas en las definiciones, algunas de las grafías selec- cionadas tienen un componente subjetivo inevitable, por lo que deben recogerse bajo un criterio de discusión no cerrada. Hemos tenido en cuenta estos aspectos en nuestras reflexiones, que no invalidan en ningún caso el debate que pretende abrir este estudio. 
Estas cabezas se concentran en varias áreas geográficas, entre las que destaca la zona pirenaica (27 UG). En la Península Ibérica sólo contabilizamos cuatro unidades gráficas y además todas ellas pertenecen al tipo animalizado. El soporte más utilizado es el parietal (34 UG). Todas las cabezas parietales, excepto dos (Rouffignac 3 y Le Gabillou 1), se encuentran en zonas profundas de la caverna. En el arte mueble tenemos $24 \mathrm{UG}$ que se grabaron sobre 20 piezas de diferente materia prima: pétrea (14 piezas), ósea (2 piezas) y 4 asta de reno. Entre la pétrea podemos distinguir 9 plaquetas, 3 cantos, 1 losa y 1 bloque; entre la ósea una costilla y un hueso indeterminado y; entre la asta dos bastones perforados y un propulsor.

La técnica más empleada es el grabado. La pintura en negro se utiliza en cuatro cabezas de la zona pirenaica. Finalmente el trazo monodigital sirvió para realizar las dos cabezas afrontadas de Rouffignac 1 y 2.

La perspectiva predominante es el perfil (41 UG). La perspectiva frontal es escasa (13 UG) y aparece sobre todo en el arte parietal de la región del Midi (Marsoulas 1 y 2, Labastide, TroisFrères 1 y 2, y Ker de Massat 2).

Las caricaturas son un fenómeno magdaleniense, y las propuestas cronológicas suelen ubicarlas en el Magdaleniense medio (37 UG). Si atendemos a estos planteamientos se puede documentar que durante la etapa anterior el fenómeno de la caricatura aparece en la Dordońa y la Gironda (6 UG), mientras que en las demás zonas este fenómeno es prácticamente inexistente. Sin embargo, durante el Magdaleniense medio este tipo de grafías surgen con fuerza en la zona pirenaica (19 UG) y en el sitio de La Marche (8 UG), mientras siguen manifestándose en la Dordońa (12 UG). En el Magdaleniense superior desaparecen en todas las zonas salvo en la pirenaica (7 UG) que continua representando estas cabezas, ahora casi siempre sobre soportes parietales.

La nariz se representa siempre salvo en una ocasión (Rouffignac 3) y es la parte anatómica más variada, detallada y compleja. Hemos reconocido ocho tipos de narices, casi todas ellas con exageraciones en el hueso nasal, el lóbulo o la punta. La más representada es una forma redonda que no existe en la naturaleza humana (20 UG) y es típica de las caricaturas.

\section{3.b) Risas y sonrisas}

Hemos reunido 31 grafías humanas que muestran trazos ascendentes en la zona de la boca (fig. 3). Se pueden distinguir tres tipos de trazos: ascendente corto o sonrisa tímida (10 UG), ascendente largo o amplia sonrisa (15 UG) y ascendente en boca abierta (6 UG), lo que puede representar la sonoridad de una risa. Las sonrisas y risas se distribuyen por cuatro regiones francesas: Midi-Pyrénées (6 UG), Aquitania (13 UG, 3 en Isturitz), Poitou-Charentes (11 UG) y Centro (1 UG), sin ningún caso claro en la Península Ibérica.

La sonrisa se representa preferiblemente sobre soporte mobiliar (20 piezas), en plaquetas pétreas (6 UG) o también astas de reno (6 UG), mediante la técnica del grabado o el esculpido. Aparece con relativa frecuencia en soportes estrechos, ya sean concebidos en posición vertical (La Garenne, Peyzie, Le Placard, La Madeleine y Gourdan 4) u horizontal (Isturitz 1 y 8, y Laugerie-Basse 1).

El arte parietal muestra un uso de la técnica diverso: el grabado (Bernifal, Combarelles, Ker de Massat y Bourdois), el trazo monodigital (Rouffignac 1 y 2), el bajo relieve (Bourdois), y la pintura en negro (Rouffignac 4, Fontanet y restos de coloración en Bourdois) y rojo (Le Portel).

Respecto al uso de la perspectiva, la sonrisa frontal — aunque es escasa - no aparece en la región pirenaica. Se expresa casi siempre de perfil y además no existe la risa frontal en el arte paleolítico. 

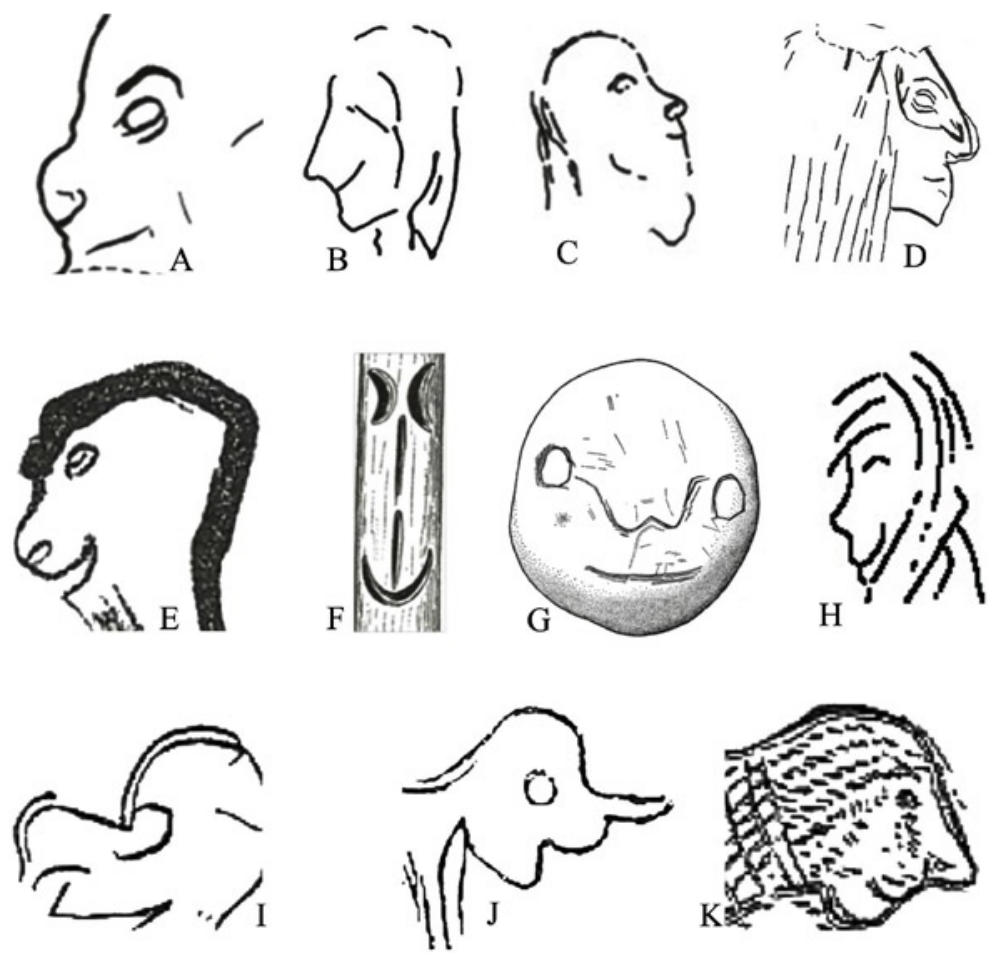

Figura 3. Sonrisas y risas (señalamos con la abreviatura $f c$ las grafias en formato completo): A Bernifal (Roussot 1986, 100, fig. 3); B Bourdois (Iakovleva, Pinçon 1997, 138, fig. 158); C La Marche (Pales, Tassin 1976, ob 9); D La Marche (Mélard 2008, 231, pl. 19. b); E (fc) Le Portel (Castillon 2004, 19, fig. 5. 1); F Roc de Marcamps (Capitan et al. 1924, 101, fig. 95. 2); G Laugerie-Basse (Roussot 1971-72, 280, fig. 1); H (fc) Gourdan (Duhard 1992, 138, fig. 4); I Isturitz (Saint-Périer 1936, 113, fig. 65); J (fc) Isturitz (Saint-Périer 1930, 93, fig. 76); K (fc) Isturitz (Saint-Périer 1936, 115, fig. 66)

Las grafías sonrientes o rientes aparecen por primera vez en yacimientos del Magdaleniense tipo navetas y azagayas Lussac-Angles. Estas grafías mobiliares se reparten en un área geográfica más o menos precisa, en un dilatado periodo de tiempo que podría situarse entre el inicio del Magdaleniense francés y el 14.280 BP del yacimiento de La Marche (Airvaux 2001, 89). Posteriormente parece que las grafías sonrientes y rientes se esparcen hacía el sur de la Dordońa y la zona pirenaica francesa, en yacimientos adscritos al Magdaleniense medio y superior/final.

Además se observan ciertas diferencias geográficas en los formatos. En la región pirenaica (incluidas las unidades del yacimiento de Isturitz) se concentran cinco de los seis casos de sonrisas o risas realizadas en figuras humanas de formato "completo» (Le Portel, Gourdan 4, Enlène 2, Isturitz 7 y 8); todas las restantes (23 UG) fueron hechas en formato cabeza. Este formato es utilizado para representar sonrisas y risas en la región aquitana (9 UG, 1 en Isturitz) y la Charente (12 UG, 9 en La Marche), lo que contrasta con la región pirenaica. 


\section{Características generales del fenómeno lúdico}

El conjunto de documentos estudiado se localiza en dos grandes zonas geo-cronológicas. La primera es una extensa área al norte de la Dordońa —en donde se constatan las primeras expresiones sonrientes - y la región del Poitou-Charentes con el yacimiento de La Marche como eje principal $^{6}$.

Y la segunda es la zona pirenaica (incluyendo Isturitz) que muestra las siguientes particularidades:

a) la cantidad de caricaturas (27 UG) y la preferencia por las de rostros humanos con rasgos anatómicos exagerados en el arte parietal del Midi (11 UG),

b) la pervivencia de las caricaturas durante el Magdaleniense superior (7 UG) mientras en el resto de áreas el fenómeno desaparece,

c) la excepcional presencia de grafías sonrientes y rientes en formato completo (5 UG) y

d) la perspectiva frontal de las caricaturas.

La zona pirenaica se nos revela como un lugar en donde el fenómeno lúdico se constata de forma relativamente abundante y particular ${ }^{7}$.

Sin embargo en la Península Ibérica no se documentan hasta la fecha sonrisas o risas evidentes y además el fenómeno de la caricatura es escaso. Se nos plantean dos posibles explicaciones: o el fenómeno lúdico en la Península Ibérica es casi inexistente o se expresó en la animalización de figuras humanas de tamaño completo ${ }^{8}$.

Las expresiones alegres se concentran en soportes mobiliares (64,5\%), y aunque los documentos son escasos y excepcionales pueden estar indicando diferencias en el carácter cotidiano de los soportes mobiliar y parietal ${ }^{9}$. El arte mueble ofrece la posibilidad de manifestarse en cualquier lugar y momento, libre de los condicionamientos arquitectónicos. Además los objetos de arte mueble se encuentran habitualmente en espacios exteriores, cercanos y accesibles a todos, y, en general, parecen ser más adecuados que los parietales ${ }^{10}$ para reflejar emociones cotidianas como la sonrisa.

La nariz muestra un variadísimo repertorio de formas y tamaños que nos induce a pensar que su ejecución estuvo basada en la "experimentación gráfica» caricatural (Gombrich 1962, 288). Los autores/as de tales cabezas representaron la nariz mediante dos procedimientos típicos de la caricatura:

${ }^{6}$ No es casualidad que un hábitat donde se desarrollaron múltiples actividades cotidianas (Mélard 2008, 144) presente el mayor número de ejemplos de nuestro repertorio (15 UG).

7 Durante este periodo circulan por la zona pirenaica algunas imágenes que han sido en ocasiones interpretadas como bromas (Péquart, Péquart 1963, 330; Laurent 1965; Delporte 1990, 152).

${ }^{8}$ La caricatura tal y como la hemos definido en el presente trabajo — reducida al formato cabeza - tiene una escasa presencia en la Península Ibérica y se limita, además, al tipo de caricatura animalizada (cuevas de Juan Gómez/Hoz, Los Casares y Abauntz). Sin embargo, casi el $60 \%$ de las grafías antropomorfas de la Península Ibérica son animalizaciones - 57 UG de las 96 UG recogidas en nuestro recopilatorio- (Lombo 2015b, 184). Cabe la posibilidad de que algunos antropomorfos peninsulares con rasgos anatómicos exagerados sean zoo-caricaturas. Se ha de tener en cuenta que la práctica de realizar personajes humanos con cabezas animales es propia de la caricatura (Wright 1875, 5-8; Vaquero 1995, 202; Pastecca 1997, 14).

${ }_{9}$ Muchos prehistoriadores han reflexionado acerca de las distinciones entre el grafismo mobiliar y rupestre (ver Barandiarán 2003, 242; 2006, 135-137).

10 El arte parietal, monumental e inmóvil (Vialou 2009, 475), parece hecho para perdurar. Desde el punto de vista gráfico parece un arte más conservador y menos dado a las innovaciones gráficas. Además las grafías cavernarias están ubicadas en espacios interiores cuyo acceso puede ser restringido (Hernando 2014, 402). 
a) la exageración de los modelos naturales existentes en la morfología humana y

b) la invención de formas nuevas en sí mismas exageradas.

Las exageraciones no pueden explicarse como el resultado de una incapacidad generalizada. Sin embargo sabemos que el ser humano está capacitado desde su más tierna infancia para reconocer el carácter cómico de las exageraciones anatómicas (Puche, Lozano 2002, 94, 95 y 112). La coincidencia entre rasgos anatómicos exagerados y expresiones alegres se detecta en ocho grafías que son a su vez clasificadas como caricaturas rientes o sonrientes.

\section{ReFLeXiones}

El término "caricatura» es un concepto moderno, pero el fenómeno gráfico que describe no lo es. El carácter de estas grafías (la variabilidad y creatividad formal, las exageraciones anatómicas - sobre todo de la nariz-, así como el formato y el tema humano) se acerca mucho al fenómeno gráfico de la caricatura que se observa en todas las culturas humanas (Alexandre 1892; Langer 2001). No obstante, hay que reconocer que el concepto de caricatura, como otros tantos conceptos aplicados a las cabezas humanas (Lombo 2015c), describen y definen el material gráfico de forma relativa. Por lo tanto, la palabra "caricatura» nos ofrece una manera de reflexionar sobre el grafismo paleolítico y puede ayudarnos a tener una visión más completa de las sociedades humanas.

La risa y la sonrisa son emociones fundamentales para el funcionamiento cotidiano de los grupos humanos (Eibl-Eibesfeldt 1993), que deben trasmitirse y perpetuarse a través de la materia gráfica. La distribución de las piezas mobiliares durante el periodo Magdaleniense puede ser un reflejo del papel de estas expresiones alegres en las relaciones intergrupales (Conkey 1980; Fritz et al. 2007; Sauvet et al. 2008; Utrilla, Bea 2008). Por otro lado, el papel del humor en el sexo, tan señalado en las culturas ágrafas (Malinowski 1971, 294-300; Apte 1985, 67-82), puede tenerse en cuenta como interpretación de los antropomorfos itifálicos de Le Portel, Laugerie-Basse 1, Gourdan 4 e Isturitz 7, o en la posible escena de acoplamiento de Enlène 2 (Bégouën et al. 1984-85, 69; Delluc 2006, 219), que presentan expresiones sonrientes o rientes. Pueden comparase, por ejemplo, con los héroes bromistas de grandes falos (Miceli 1984) de las mitologías polinesias (Campbell 1991, 227) o sioux (Radin 1965, 15).

El estudio del humor en Prehistoria puede y debe favorecer el diálogo y la reflexión sin menospreciar otras posibilidades interpretativas que posibiliten un conocimiento más profundo de nuestra naturaleza humana. Estudiamos el arte desprovisto de emociones. Los ejemplos seleccionados en este trabajo son la expresión cultural de un estado anímico fundamental para el funcionamiento de la vida cotidiana de las poblaciones ágrafas. La alegría es una poderosa fuerza creadora ${ }^{11}$ entre los pueblos cazadores-recolectores (Bowra 1984, 285) cuya influencia no ha sido, salvo excepciones (Patte 1960, 37), suficientemente bien estudiada en el arte rupestre.

El misterio de la risa cautiva por su fuerza y vitalidad, otorga sentido a las ocupaciones diarias y a los placeres cotidianos. Sin estas distracciones de lo serio, sin estas manifestaciones alegres de la vida, el ser humano no puede afrontar los quehaceres de la rutina, ni la propia condición de su efímera existencia de la que es consciente.

11 Por ejemplo los cantos de burla o risa de los esquimales (Bowra 1984, 36, 148 y 172), o los mitos sobre la risa entre los indígenas sudamericanos (LéviStrauss 1968, 92, 96, 112, 113, 123). 
Las sonrisas, risas y caricaturas paleolíticas puede que sean la expresión de un «humor prehistórico» que facilitó la transmisión de un estado de ánimo sin el cual no hubiéramos podido sobrevivir como especie ${ }^{12}$.

Anexo 1

\begin{tabular}{|c|c|c|}
\hline LUGAR & ClasificaCión & BIBLIOGRAFÍA \\
\hline Bourdois & Sonrisa & Iakovleva, Pinçon 1997, fig. 6 \\
\hline Marche 1 & Sonrisa & Pales, Tassin 1976, ob. 33 \\
\hline Marche 2 & Caricatura & Airvaux 2001, 95, fig. 69 \\
\hline Marche 3 & Caricatura & Pales, Tassin 1976, ob. 12, pl. 24 \\
\hline Marche 4 & Caricatura & Pales, Tassin 1976, ob. 16, pl. 29 \\
\hline Marche 5 & Caricatura/sonrisa & Mélard 2008, 231, pl. 19. b \\
\hline Marche 6 & Caricatura & Pales, Tassin 1976: ob. 2, pl. 61 \\
\hline Marche 7 & Caricatura & Pales, Tassin 1976, ob. 6, pl. 15 \\
\hline Marche 8 & Caricatura & Mélard 2008, 162, fig. 18 \\
\hline Marche 9 & Sonrisa & Pales, Tassin 1976, ob 9, pl. 19 \\
\hline Marche 10 & Sonrisa & Pales, Tassin 1976, ob. 30 , pl. 75 \\
\hline Marche 11 & Sonrisa & Pales, Tassin 1976, ob. 7, pl. 16 \\
\hline Marche 12 & Sonrisa & Pales, Tassin 1976, ob. 25, pl. 52 \\
\hline Marche 13 & Risa & Pales, Tassin 1976, ob. 29, pl. 67a \\
\hline Marche 14 & Sonrisa & Pales, Tassin 1976, ob. 2, pl. 6 \\
\hline Marche 15 & Sonrisa & Mélard 2008, 162, fig. 18, pl. 55c \\
\hline Garenne & Sonrisa & Fuentes 2013, 996, fig. 11a \\
\hline Placard & Sonrisa & Laurent 1963, 564, fig. 1 \\
\hline Peyzie & Sonrisa & Allain et al. 1985, 93, fig. 64.1 \\
\hline Gabillou 1 & Animalización & Gaussen 1964, pl. 34, fig. 1 \\
\hline Gabillou 2 & Caricatura & Gaussen 1964, pl. 8, fig. 3 \\
\hline Rouffignac 1 & Caricatura/risa & Barrière 1982, 142, fig. 43 \\
\hline Rouffignac 2 & Caricatura & Barrière 1982, 142, fig. 43 \\
\hline Rouffignac 3 & Animalización & Barrière 1982, 16, fig. 11 \\
\hline Rouffignac 4 & Caricatura/sonrisa & Barrière 1984, 204, fig. 6 \\
\hline Saint-Cirq 1 & Animalización & Delluc et al. 1987, 388, fig. 33 \\
\hline Saint-Cirq 2 & Animalización & Delluc et al. 1987, 386, fig. 28 \\
\hline Combarelles 1 & Animalización & Barrière 1997, 212, fig. 169 \\
\hline Combarelles 2 & Caricatura & Barrière 1997, 97 \\
\hline Combarelles 3 & Caricatura & Archambeau, Archambeau 1991, 73, fig. 32 \\
\hline Combarelles 4 & Animalización & Barrière 1997, 76 \\
\hline Combarelles 5 & Animalización & Barrière 1997, 389, fig. 423 \\
\hline Combarelles 6 & Caricatura & Barrière 1997, 411, fig. 463 \\
\hline
\end{tabular}

12 Agradezco a Pilar Utrilla y Manuel Bea que dirigieron mi tesis. A José Luis Sanchidrián, María Ángeles Medina, José María Cobos y Sonia Cosano por su

alegre amistad. A Ignacio Barandiarán y Marcos García Diez por haberme dado la oportunidad de participar en este monográfico. 


\begin{tabular}{|c|c|c|}
\hline LUGAR & ClasificaCión & BibLIOGRAFía \\
\hline Combarelles 7 & Animalización & Barrière 1997, 389, fig. 426 \\
\hline Combarelles 8 & Sonrisa & Barrière 1997, 249, fig. 223 \\
\hline Bernifal & Sonrisa & Roussot 1986, 100, fig. 3 \\
\hline Laugerie-Basse 1 & Sonrisa & Pales, Tassin 1976, 80, fig. 27 \\
\hline Laugerie-Basse 2 & Sonrisa & Roussot 1971-72, 280, fig. 1 \\
\hline Madeleine & Sonrisa & Capitan et al. 1924, 108, fig. 100.6 \\
\hline Péchialet & Caricatura & Breuil 1927, 107, fig. 3 \\
\hline Marcamps 1 & Sonrisa & Capitan et al. 1924, 101, fig. 95.2 \\
\hline Marcamps 2 & Caricatura/sonrisa & Barandiarán 2006, 180, fig. 69 \\
\hline Marcamps 3 & Caricatura & Barandiarán 2006, 180, fig. 69 \\
\hline Marcamps 4 & Caricatura & Barandiarán 2006, 180, fig. 69 \\
\hline Trois-Frères 1 & Caricatura & Bégouën, Breuil 1958, 35 fig. 39 \\
\hline Trois-Frères 2 & Animalización & Bégouën, Breuil, 1958, 35 fig. 39 \\
\hline Trois-Frères 3 & Animalización & Bégouën et al. 2014, 133, fig. 114 \\
\hline Trois-Frères 4 & Caricatura & Bégouën, Breuil 1958, 72, figs. 75-77 \\
\hline Mas d'Azil & Caricatura & Castillon 2004, 17, fig. 4 \\
\hline Fontanet 1 & Caricatura/sonrisa & Clottes et al. 1984 , 434, fig. 2 \\
\hline Fontanet 2 & Caricatura & Clottes et al. 1984, 434, fig. 2 \\
\hline Le Portel & Risa & Castillon 2004,19 , fig. 5.1 y 2 \\
\hline Massat 1 & Animalización/risa & Barrière 1990, 35, fig. 15 \\
\hline Massat 2 & Caricatura & Barrière 1990, 66, fig. 51 \\
\hline Massat 3 & Caricatura & Barrière 1990, 64, fig. 48 \\
\hline Massat 4 & Caricatura & Barrière 1990, fig. 50 \\
\hline Marsoulas 1 & Caricatura & Leroi-Gourhan 1971, 397 \\
\hline Marsoulas 2 & Caricatura & Vialou 1986, 212, fig. 165 \\
\hline Labastide & Caricatura & Omnes 1984,22 , pl. 3 \\
\hline Erberua & Caricatura & Garate, Bourrillon 2009, 66, fig. 5.8 \\
\hline Sinhikole & Animalización & Garate, Bourrillon 2009, 66, fig. 5.1 \\
\hline Enlène 1 & Caricatura/sonrisa & Bégouën, Clottes 1995, 34, fig. 3 \\
\hline Enlène 2 & Sonrisa & Bégouën et al. 1984-85, 68, fig. 66 \\
\hline Bédeilhac & Caricatura & Sieveking 1987, 175, fig. 38 \\
\hline Gourdan 1 & Animalización & Capitan et al. 1924, 99, fig. 93 \\
\hline Gourdan 2 y 3 & Caricaturas & Barandiarán 2006, 103, fig. 42 \\
\hline Gourdan 4 & Sonrisa & Duhard 1992, 138, fig. 4 \\
\hline Isturitz 1 & Caricatura/risa & Saint-Périer 1936, 113, fig. 65 \\
\hline Isturitz 2,3 y 4 & Caricaturas & Saint-Périer, 1936: 113, fig. 65 \\
\hline Isturitz 5 & Caricatura & Saint-Périer 1930, 93, fig. 76.2 \\
\hline Isturitz 6 & Caricatura & Saint-Périer 1930, 93, fig. 76.1 \\
\hline Isturitz 7 & Risa & Saint-Périer 1930, 93, fig. 76 \\
\hline Isturitz 8 & Sonrisa & Saint-Périer 1936, 115, fig. 66 \\
\hline Colombier & Caricatura & Vialou 1998,88 \\
\hline Juan Gómez/Hoz & Animalización & Barandiarán et al. 1981, 125, fig. 3 \\
\hline Casares 1 y 2 & Animalización & Balbín, Alcolea 1992, 420, fig. 24 \\
\hline Abauntz & Animalización & Utrilla et al. 2007-2008, 238, fig. 3 \\
\hline
\end{tabular}




\section{BibliografíA}

Airvaux, J., 2001, L'art préhistorique du Poitou-Charentes. Sculptures et gravures des temps glaciaires, Paris: La maison des roches.

Alexandre, A., 1892, L'Art du rire et de la caricature, Paris: Ancienne Maison Quantin. Librairies-Imprimeries Réunies.

Allain, J., Desbrosse Rene, K., Kozlowski, J., Rigaud, A., Jeannet, M., Leroi-Gourhan, A., 1985, «Le Magdalénien à navettes», Gallia préhistoire 28 (1), 37-124.

Alpert, B.O., 1992, «Des preuves de sens ludique dans l'art au Pléistocène Supérieur», L'Anthropologie 96 (2-3), 219-244.

—, 2008, The Creative Ice Age Brain: Cave Art in the Light of Neuroscience, New York: Foundation 20/21.

Apte, M.L., 1985, Humor and Laughter: An Anthropological Approach, Hardcover: Cornell University Press.

Archambeau, M., Archambeau, C., 1991, «Les figurations humaines pariétales de la grotte des Combarelles», Gallia Préhistoire 33, 53-81.

Bahn, P.G., Vertut, J., 1988, Images of the Ice Age, London: Windward.

Balbín Behrmann, R., Alcolea González, J. J., 1992, "La grotte de los Casares et l'art paléolitique de la Meseta espagnole», L'Anthropologie 96 (2/3), 397-452.

Barandiarán, I., 2003, Grupos homoespecificos en el imaginario mobiliar magdaleniense. Retratos de familia y cuadros de género, [Anejos de Veleia. Series minor 21], Vitoria-Gasteiz: Universidad del País Vasco.

—, 2006, Imágenes y adornos en el arte portátil paleolítico, Barcelona: Ariel Prehistoria.

Barandiarán, I., González Echegaray, J., González Cuadra, F., 1981, "Grabados de la cueva de La Hoz (Sámano; prov. Santander)», en: Altamira Symposium, Madrid: Ministerio de Cultura, 119-128.

BARLEY, N., 2010, El antropólogo inocente, Barcelona: Anagrama.

BARrière, C., 1982, L'art pariétal de Rouffignac. La grotte aux cents mammouths, [Mémoire IV de l'Institut d'Art Préhistorique de Toulouse/Fondation Singer-Polignac], Paris: Éditions Picard.

—, 1990, L'art pariétal du Ker de Massat, Toulouse: Presses Universitaires du Mirail.

—, 1997, L'art pariétal des grottes les Combarelles, Angoulême: AMPRA/PALEO.

Beaudet, J.-M., 1996, «Rire. Un exemple d'Amazonie», L'Homme 140, 81-99.

BEAUNE DE S., 2007, «La préhistoire est-elle toujours une science humaine?», en: J. Évin, J. (dir.), Un siècle de construction du discours scientifique en Préhistoire, vol. III "Aux conceptions d'aujourd'hui», Paris: Mémoires de la Société préhistorique française, 13-21.

BÉgouën, R., Breuil, H., 1958, Les cavernes du Volp. Trois-Frères. Tuc d'Audoubert, Paris: Arts et Métiers Graphiques.

Bégouën, R., Briois, F., Clottes, J., Servelle, C., 1984-85, «Art mobiler sur support lithique d'Enlène (Montesquieu-Avantès, Ariège)», Ars Praehistorica III/IV, 25-80.

BÉgouën, R., Clottes, J., 1995, «Les humains dans les cavernes du Volp», en H. Delporte (ed.), La Dame de Brassempouy, [ERAULT 74], Liège: Etudes et Recherches Archéologiques de l'Université de Liège, 29-40.

Bégouën, R., Clottes, J., Feruglio, V., Pastoors, A., 2014, La caverne de Trois-Frères. Anthologie d'un exceptionnel sanctuaire préhistorique. Paris: Somogy Éditions d'art. Association Louis Bégouën.

Bowra, C.M., 1984, Poesía y canto primitivo, Barcelona: Antoni Bosch.

Brackelaire, J.-L., 1993, "Changer pour rire. Les relations de plaisanterie des Tarahumaras: figure et mesure du changement», Anthropologie et Sociétés 17 (3), 125-140.

Breuil, H., 1927, «Euvres d'art paléolithiques inédites du Périgord et art oriental d'Espagne», Revue Anthropologique 38, 103-104.

Cabré Aguiló, J., 1940-1941, "Figuras antropomorfas de la cueva de los Casares (Guadalajara)», Archivo Español de Arqueología 14, 81-97.

Campbell, J., 1991, Las máscaras de Dios: Mitología primitiva, Madrid: Alianza.

Capitan, L., Breuil, H., Peyrony, D., 1924, Les Combarelles aux Eyzies (Dordogne), Paris: Masson et Cie. 
Cartailhac, E., Breuil, H., 1906, La caverne d'Altamira à Santillane, près Santander (Espagne), Monaco: Imprimerie de Monaco.

Castillon, R., 2004, "Quelques figures à la grotte du Portel», Bulletin Préhistoire du Sud-Ouest 11 (1), $5-21$.

Clemen, C., 1932, Urgeschichtliche Religion: Die Religion der Stein-, Bronze- und Eisenzeit, Bonn: Ludwig Röhrscheid Verlag.

Clottes, J., Rouzaud, F., Wahl, L., 1984, "Grotte de Fontanet», en: A. Leroi-Gourhan (ed.), L'art des Cavernes. Atlas des grottes ornées paléolithiques françaises, Paris: Ministère de la culture. Imprimerie Nationale, 433-437.

Condominas, G., 1991, Lo exótico es cotidiano, Madrid: Ediciones Júcar.

Conkey, M., 1980, "The identification of prehistoric hunter-gatherer aggregation sites: the case of Altamire», Curren Anthropology 21, 609-630.

DARwIn, C., 1984 [1872], La expresión de las emociones en los animales y en el hombre, Madrid: Alianza Editorial.

Davila, R.M., Owren, M.J., Zimmermann, E., 2009, «Reconstructing the evolution of laughter in great apes and humans", Current Biology, 19: 1106-1111.

Delluc, G., 2006, Le sexe au temps des Cro-Magnons, Périgeux : Piltote 24.

Delluc, B., Delluc, G., 2009, "Art paléolithique en Périgord. Les représentations humaines pariétales», L'Anthropologie 113, 629-661.

Delluc, B., Delluc, G., Guichard, F., 1987, «La grotte ornée de Saint-Cirq (Dordogne)», Bulletin de la Société préhistorique Françoise 84 (10/12), 364-393.

Delporte, H., 1990, L'image des animaux dans l'art préhistorique, Paris: Picard.

Driessen, H., 1999, "Humor, risa y trabajo de campo: apuntes desde la antropología», en: J. Bremmer, H. Roodenburg (coord.), Una historia cultural del humor. Desde la Antigüedad hasta nuestros dias, Madrid: Sequitur, 227-246.

Duhard, J.-P., 1992, "Les humains ithyphalliques dans l'art paléolithique», Bulletin de la Société Préhistorique de l'Ariège-Pyrénées XLVII, 133-159.

—, 1996, Réalisme de l’image masculine paléolithique, Grenoble: Jérôme Millon.

Dunbar, 1996, Grooming, Gossip, and the Evolution of Language, London: Faber and Faber.

Evans-Pritchard, E.E., 1988, Las teorias de la religión primitiva, Madrid: Siglo veintiuno.

Fiore, D., 2014, «Archaeology of Art: Theoretical Frameworks», en: C. Smith (ed.), Enciclopedia of Global Archaeology, Heidelberg: Springer, 436-449.

Forrer, R., 1908, Urgeschichte des Europäers: von der Menschwerdung bis zum Anbruch der Geschichte, Stuttgart: W. Spemann.

Fraguas Bravo, A., 2007, Del panel a la hegemonia: nuevas teorías y tecnologías para el arte rupestre del noreste de Africa, Madrid: Tesis doctoral inédita.

Fritz, C., Tosello, G., Sauvet, G., 2007, «Groupes ethniques, territoires, échanges: La "notion de frontière” dans l'art magdalénien», en : N. Cazals, J. González Urquijo, X. Terradas (eds.), Frontières naturelles et frontières culturelles dans les Pyrénées préhistoriques [ Monografías del IIIPC], Santander: Publican ediciones, 164-181.

Fuentes, O., 2010, "Les représentations humaines au magdalénien en Poitou-Charentes», en: J. BuissonCatil, J. Primault (eds.), Préhistoire entre Vienne et Charente. Hommes et sociétés du Paléolithique, [Mémoire XXXVIII], Association des Publications Chauvinoises, 383-396.

—, 2013, "The depiction of the individual in prehistory: human representations in Magdalenian societies», Antiquity 87, 985-1000.

Gailli, R., 1980, «Anthropomorphes extraordinaires de la grotte du Ker de Massat Ariège», Caesaraugusta 51-52, 23-37.

Garate, D., Bourrillon, R., 2009, «Les grottes ornées du massif des Arbailles (Pyrénées-Atlantiques) dans le contexte artistique du Tardiglaciaire», Bulletin de la Société Préhistorique Ariège-Pyrénées LXIV, 61-72.

Gaussen, J., 1964, La grotte ornée de Gabillou (Près Mussidan, Dordogne), Bordeaux: Delmas. 
Gombrich, E.H., 1962, «The Experiment of Caricature», en: Art and Illusion A study in the psychology of pictorial representation, London: Phaidon Press, 279-303.

Guthrie, R.D., 2005, The Nature of Paleolithic Art, Chicago and London: The University of Chicago Press.

Halverson, J., 1987, "Art for Art's Sake in the Paleolithic», Current Anthropology 28 (1), 63-71.

Наму, E.-T., 1908, «La figure humaine chez le sauvage et chez l'enfant», L'Anthropologie 19, 385-407.

Hernando Álvarez, C., 2014, La sociedad a través del arte: las tradiciones gráficas premagdalenienses en la región cantábrica, Salamanca: Tesis doctoral inédita.

Huizinga, J., 1968, Homo ludens, Madrid: Alianza.

Iakovleva, L., Pinçon, G., 1997, La frise sculptée du Roc-aux-Sorciers, Réunion des musées nationaux, Paris: Réunion des Musées Nationaux.

Langner, M., 2001, Antike Graffitizeichnungen. Motive, Gestaltung und Bedeutung. Wiesbaden: Reichert.

Laurent, P., 1963, "La tête humaine gravée sur bois de renne de la grotte du Placard (Charente)", L'Anthropologie 67, 563-569.

—, 1965, Heureuse Préhistoire, Périgueux: Pierre Fanlac.

Leonardi, P., 1989, Sacralità, arte e grafia paleolitiche: splendori e problemi, Calliano: Museo civico di storia naturale di Trieste.

Leroi-Gourhan, A., 1971, Préhistoire de l'Art Occidental, Paris: Éditions d'Art Lucien Mazenod.

—, 1984, Simbolos, artes y creencias de la Prehistoria, Madrid: Istmo.

LÉvi-Strauss, C., 1968, Mitológicas I. Lo crudo y lo cocido, México: Fondo de Cultura Económica.

Lombo Montañés, A., 2013, «El concepto de caricatura en el arte paleolítico y los graffiti actuales», Arkeogazte 3, 243-270.

—, 2014, «Interpretación de retruécanos, imágenes de doble sentido y bromas en el arte paleolítico», Complutum 25 (1), 35-46.

—, 2015a, «Los significados del arte paleolítico: una revisión historiográfica y crítica», ArqueoWeb 16, 4-20.

—, 2015b, Risas, sonrisas y caricaturas en las manifestaciones gráficas paleoliticas, Zaragoza: Tesis doctoral inédita.

—, 2015c, "Grotescos, máscaras y fantômes en el arte paleolítico», Pyrenae 46 (2), 7-29.

LuQuet, G.H., 1910, «Sur les caractères des figures humaines dans l'art paléolithique», L'Anthropologie 21, 409-423.

—, 1926, L'art et la religion des hommes fossiles, Paris: Masson et Cie Editeurs.

Malinowski, B., 1971, La vida sexual de los salvajes del Noroeste de Melanesia, Madrid: Morata.

Marshall, L., 1961, «Sharing, Talking, and Giving: Relief of Social Tensions Among Kung Bushmen», Africa 31 (3), 231-249.

MéLARD, N., 2008, «Pierres gravées de la Marche à Lussac-Les-Châteaux (Vienne). Techniques, technologie et interprétations», Gallia Préhistoire 50, 143-268.

Miceli, S., 1984, Il demiurgo trasgressivo. Studio sul trickster, Palermo: Sellerio.

Mithen, S., 1998, Arqueología de la mente. Origenes del arte, de la religión y de la ciencia, Barcelona: Crítica Grijalbo Mondadori.

Omnes, J., 1984, «Le sanctuaire magdalénien de la grotte de Labastide (Hautes-Pyrénées. France)», Munibe $36,19-26$.

Pales, L., Tassin de Saint Pereuse, M., 1976, Les gravures de La Marche. II. Les Humains, Paris: Éditions Ophrys.

Pastecca, 1997, Dibujando caricaturas, Barcelona: Grupo Editorial Ceac.

Patte, E., 1960, Les Hommes Préhistoriques et la Religion, Paris: A. Et Picard et Cie.

PÉquart, M., PÉQuart, S.-J., 1963, «Grotte du Mas d'Azil (Ariège). Une nouvelle galerie magdalénienne», Annales de Paléontologie 49, 257-351.

Piette, E., 1907, L'art pendant l'Âge du Renne. Album de cents planches dessinées par J. Pilloy, Paris: Masson et Cie.

Plassard, J., 1999, Rouffignac. Le sanctuaire des mammouths, Paris: Éditions du Seuil. 
Provine, R., 2000, Laughther. A scientific investigation, London: Faber and Faber.

Puche Navarro, R., Lozano Hormaza H., 2002, El sentido del humor en el niño: estudio empírico, Bogotá: Siglo del Hombre Editores.

RadCLIFfe-Brown, A.R., 1940, «On Joking Relationships», Journal of the International African Institute 13 (3): 195-210.

Radin, P., 1969, The Trickster: A Study in American Indian Mythology, New York: Greenwood Press.

Richard, N., 1993, «De l'art ludique a l'art magique. Interprétations de l'art pariétal au XIX siècle», Bulletin de la Société Préhistorique Française 90 (1-2), 60-68.

Ridell, W. H., 1940, «Dead or Alive», Antiquity 14, 158-162.

Ripoll, E., 1957-1958, «Las representaciones antropomorfas en el arte paleolítico español», Ampurias 1920, 167-192.

Roussot, A., 1986, «La figuration humaine de Bernifal (Dordogne)», en: Estudios en Homenaje al Dr. Antonio Beltrán Martínez, Zaragoza: Facultad de Filosofía y Letras, 93-102.

—, 1971-1972, «El desconocido de Laugerie-Basse», Ampurias 33-34, 279-281.

Saccasyn della Santa, E., 1947, Les figures humaines du Paléolithique supérieur euroasiatique, Anvers: De Sikkel.

Saint-PÉrier, R. DE, 1930, La grotte d'Isturitz I. Le magdalénien de la salle de Saint-Martin, [Archives de l'Institut Paléontologie Humaine mémoire 7], Paris: Masson et Cie Éditeurs.

—, 1936, La grotte d'Isturitz II. Le Magdalénien de la Grande Salle, [Archives de l'Institut Paléontologie Humaine mémoire 17], Paris: Masson et Cie Éditeurs.

Sanchidrián, J.L., 2001, Manual de Arte Prehistórico, Barcelona: Ariel Prehistoria.

Sauvet, G., Fortea, J. Fritz, C. Tosello, G., 2008, "Crónica de los intercambios entre los grupos humanos paleolíticos. La contribución del arte para el periodo 20000-12000 años BP», Zephyrus LXI, 3359.

Schiller, J.C.F., 1981, [1773-1774], Cartas sobre la educación estética del hombre, Aguilar: Buenos Aires.

Sievering, A., 1987, Engraved Magdalenian Plaquettes. A regional and stylistic analysis of Stone, bone and antler plaquettes from Upper Palaeolithic site in France and Cantabric Spain. [BAR International Series 369], Oxford: Archaeopress.

SinUÉS DEL VAL, M., 2010, «1864-1903: progreso, academicismo y arte prehistórico. Sustrato sociocultural del arte lúdico", Cuadernos de Arqueología de la Universidad de Navarra 18, 63-110.

Tymula, S., 1995, «Figures composites de l'art paléolithique européen», Paléo 7, 211-227.

Utrilla, P., Bea, M., 2008, "Santuaires rupestres comme marqueurs d'identité territoriale: Sites d'agrégation et animaux "sacrés" ", Bulletin de la Société Préhistorique de l'Ariège-Pyrénées 63, 109-133.

Utrilla, P., Mazo, C., Sopena, M.C., Domingo, R., Martínez-Bea, M., 2007-2008, «Ríos, montañas y charcas: una representación de paisaje en el bloque 1 de la cueva de Abauntz», Veleia 24-25, 229-260.

Vaquero Turcios, J., 1995, Maestros subterráneos, Madrid: Celeste Ediciones.

Verworn, M., 1908, Zur Psychologie der primitiven Kunst, Jena: Fischer.

Vialou, D., 1986, L'art des grottes en Ariège magdalénien. XXIle supplément à Gallia Préhistoire, Paris: Éditions du C.N.R.S.

—, 1998, L'art des grottes, Paris: Éditions Scala.

—, 2009, "L'image du sens, en préhistoire», L'Anthropologie 113, 464-477.

Wright, T., 1875, A History of Caricature and Grotesque in Literature and Art, London: Chatto and Windus. 